EPJ manuscript No.

(will be inserted by the editor)

\title{
$K \bar{K}$ photoproduction from protons
}

\author{
A. Sibirtsev ${ }^{1}$, J. Haidenbauer ${ }^{2}$, S. Krewald ${ }^{1}$, U.-G. Meißner ${ }^{1,2}$ and A.W. Thomas ${ }^{3}$ \\ 1 Helmholtz-Institut für Strahlen- und Kernphysik (Theorie), Universität Bonn, Nußallee 14-16, D-53115 Bonn, Germany \\ 2 Institut für Kernphysik (Theorie), Forschungszentrum Jülich, D-52425 Jülich, Germany \\ 3 Jefferson Lab, 12000 Jefferson Ave., Newport News, VA 23606, USA
}

Received: date / Revised version: date

\begin{abstract}
We study the contribution of the Drell mechanism driven by $K^{+}$and $K^{-}$exchange to the reaction $\gamma N \rightarrow K \bar{K} N$. Our calculation implements the full $K N$ and $\bar{K} N$ reaction amplitudes in the form of partial wave amplitudes taken from a meson-exchange model $(K N)$ and a partial wave analysis $(\bar{K} N)$, respectively. Comparing our results to data of the LAMP2 collaboration we observe that the Drell mechanism alone cannot describe the large $\Lambda(1520)$ photoproduction rate observed experimentally. We argue that the discrepancy could be due to significant contributions from $K^{*}$ meson exchange with subsequent excitation of the $\Lambda(1520)$ resonance. After adding such contributions to our model a good agreement of the LAMP2 experiment is achieved. When applying the same model to the recent SAPHIR data we find an excellent description of the $K^{+} p$ spectrum and we find evidence for a hyperon resonance with $M_{R}=1617 \pm 2 \mathrm{MeV}$ and $\Gamma_{R}=117 \pm 4 \mathrm{MeV}$ in the $K^{-} p$ mass distribution.
\end{abstract}

PACS. 11.80.-m Relativistic scattering theory - 11.80-Et Partial-wave analysis - 12.40.Nn Regge theory, duality, absorptive/optical models - 13.60.Le Meson production - 13.60.Rj Baryon production - 13.75.Jz Kaon-baryon interactions

\section{Introduction}

The reaction $\gamma N \rightarrow K \bar{K} N$ offers an excellent opportunity for hadronic spectroscopy. First of all, it allows access to the $K N$ system, a channel which has received much attention recently because the exotic $\Theta^{+}(1540)$ pentaquark couples to it. With regard to that issue experiments were performed at SPring -8 , CEBAF and ELSA using either a free or bound target nucleon [1-4]. The $\bar{K} N$ system can serve as a source for hyperon resonance spectroscopy. In fact, the most recent data on the $\Lambda(1520)$ hyperon quoted by PDG [5] were obtained by the LAMP2 group [6] from the reaction $\gamma p \rightarrow K^{+} K^{-} p$ at photon energies $2.8 \leq$ $E_{\gamma} \leq 4.8 \mathrm{GeV}$. Moreover, the missing mass spectrum measured in $\gamma p \rightarrow K^{+} X$ at $E_{\gamma}=11 \mathrm{GeV}$ [7] indicates many $\Lambda$ and $\Sigma$ resonances and clearly illustrates the promising perspectives of the reaction $\gamma N \rightarrow K \bar{K} N$ with respect to hyperon spectroscopy. Finally, the $K \bar{K}$ system allows one to investigate mesonic resonances with normal and exotic quantum numbers. Among those are the light scalar mesons $f_{0}(980)$ and $a_{0}(980)$ whose nature is still under debate [8-10].

The different baryonic and mesonic resonances that can occur in the reaction $\gamma N \rightarrow K \bar{K} N$ induce a considerable complexibility in the overall data analysis. In particular, possible kinematical reflections, but also momenta and angular cuts of the final state due to a limited detector acceptance might generate resonance-like structures [11], which do not correspond to genuine physical quantities. Thus, it would be rather useful to construct a phenomenological model that allows to control and constrain the background as much as possible. Thereby, it is crucial that one includes not only "true" background contributions but also available experimental information on already well established baryonic and mesonic resonances that may contribute to the final state. Indeed, a similar strategy was followed by Drell [12], Söding [13] and Krass [14] for the analysis of the reaction $\gamma p \rightarrow \pi^{+} \pi^{-} p$ already a long time ago. In particular, Söding [13] suggested that one should construct a model that incorporates the Drell mechanism using available experimental information for $\pi N \rightarrow \pi N$ elastic scattering and then apply this model to the $\gamma N \rightarrow \pi \pi N$ reaction to search for new resonances or new phenomena $[15,16]$.

The principal aim of our work is to develop a model for the background to the reaction $\gamma N \rightarrow K \bar{K} N$ by incorporating, as far as possible, all presently available and well established experimental results. To be more concrete, in the present paper we consider the Drell mechanism for the reaction $\gamma N \rightarrow K \bar{K} N$. This allows us to take into account the experimental knowledge of the reaction amplitudes in the $K N$ and $\bar{K} N$ subsystems. Specifically, we can naturally account for the manifestation of resonances in the $\bar{K} N$ and $K N$ channels. For the former this concerns, in particular, the $\Lambda(1520)$ resonance. The results of our calculation are then compared with available experimental data on photoproduction of the $\Lambda(1520)$ and specifically with the $K^{+} p$ and $K^{-} p$ invariant mass spectra reported by Barber et al. [6].

Within the last year or so several papers have appeared that deal with the reaction $\gamma N \rightarrow K \bar{K} N$ and/or the photoproduction of the $\Lambda(1520)$ hyperon [17-23]. Most of those investigations were driven by the quest for the $\Theta^{+}(1540)$ pen- 
taquark. Though a large variety of reaction mechanisms were considered none of those studies takes into account the Drell mechanism based on the full $K N$ and $\bar{K} N$ amplitudes. In fact, in some works $K$ exchange is considered but then the $K N$ and/or $\bar{K} N$ amplitudes are approximated by tree-level resonance and/or t-channel meson-exchange diagrams [19,23].

Therefore, our present paper is complementary to those other studies and, moreover, it has the potential to provide additional and important information on the photoproduction of the $K \bar{K}$ system. Specifically, it allows for a reliable evaluation of the background contribution due to $K$ exchange that is present in all such photon induced reactions and thus should be taken into account in the analysis of experimental results.

It will be shown in our analysis that the Drell mechanism alone is not sufficient to explain the $K^{+} p$ and $K^{-} p$ invariant mass spectra measured by the LAMP2 group [6]. Specifically the latter is significantly underestimated in the region of the $\Lambda(1520)$ peak. This implies that the $\Lambda(1520)$ photoproduction cross section is much larger than in corresponding hadron induced reactions. We then consider $K^{*}$ meson exchange in conjunction with the excitation of the $1(1520)$ resonance as additional reaction mechanism. Within such a scenario it is indeed possible to achieve a satisfactory description of the large $\Lambda(1520)$ photoproduction rate as reflected in the $K^{-} p$ mass spectrum [6].

The paper is organized as follows. In Section 2 we formulate our model to calculate the Drell mechanism. In particular we specify the $K N$ and $\bar{K} N$ amplitudes that we employ in our investigation. Section 3 presents a comparison between available data on $K^{+} p$ and $K^{-} p$ invariant mass spectra from the reaction $\gamma N \rightarrow K \bar{K} N$ with our results based on the Drell mechanism. We also consider additional contributions which we assume to be due to $K^{*}$ meson exchange. In Section 4 we apply our model to the recent SAPHIR data for $\gamma p \rightarrow K^{+} K^{-} p$. We demonstrate that from these data one can indeed deduce the parameters of a hyperon resonance, probably the $\Lambda(1600)$. A comparision with new data from the CLAS Collaboration is presented in Section 4. We summarize our results with our Conclusion.

\section{The Drell mechanism and the $\bar{K} N$ reaction}

The Drell mechanism for the reaction $\gamma N \rightarrow K \bar{K} N$ is shown by the two diagrams a) and b) in Fig. 1. Only the exchanges of charged kaons $K^{+}$and $K^{-}$contribute to the reaction, since photons do not couple to neutral $K$ mesons. However, the rescattering amplitude includes both elastic scattering and charge exchange. The amplitude for $t$-channel $K^{-}$exchange is given as [12-14]

$$
\mathcal{M}_{K^{-}}=-2 \boldsymbol{\epsilon}_{\gamma} \cdot \boldsymbol{q}_{1} \frac{e T_{K^{-} p}\left(s_{2}, t_{2}\right) F\left(t_{1}\right)}{t_{1}-m_{K}^{2}},
$$

where $\boldsymbol{\epsilon}_{\gamma}$ is the photon polarization vector, $\boldsymbol{q}_{1}$ is the momentum of the $K^{+}$meson in the $\gamma N$ c.m. system, $t_{1}$ is the squared fourmomentum transferred between the photon and the $K^{+}$meson and $T_{K^{-} p}$ is the $K^{-} p$ scattering or charge exchange amplitude, which depends on the squared invariant mass $s_{2}$ of the $K^{-} p$
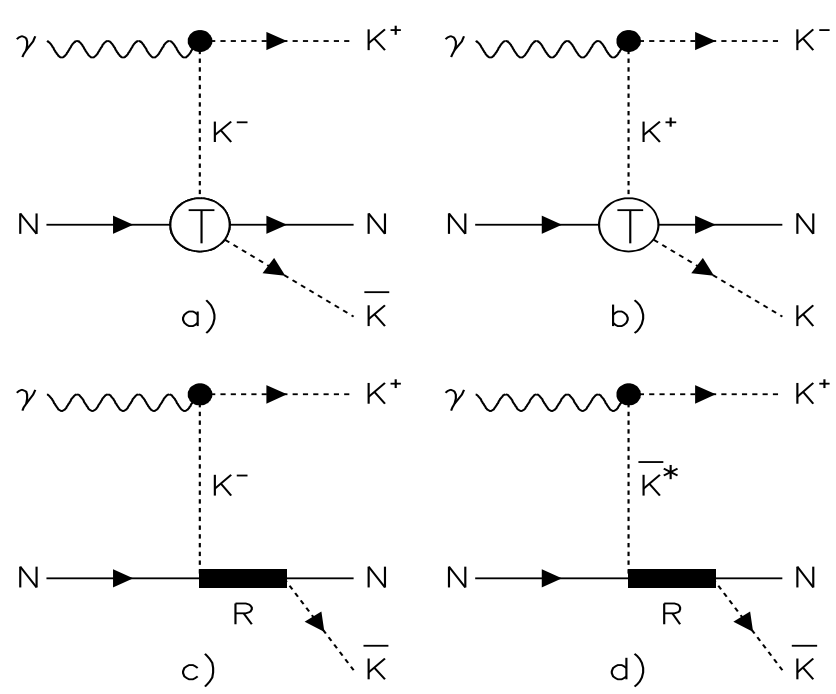

Fig. 1. Diagrams for the reactions $\gamma N \rightarrow K \bar{K} N$. The Drell mechanism for $K$ exchange with full $\bar{K} N$ and $K N$ resonant and nonresonant amplitudes is shown by a) and b). Diagrams c) and d) show $K$ and $K^{*}$ exchanges through the excitation of $Y$ hyperon resonances only. Note that in the Drell mechanism the diagram c) is naturally included in the process a).

system and the squared four-momentum $t_{2}$ transferred from the initial to the final proton. Furthermore, $F$ is a form factor that accounts for the offshellness of the $K^{-} p$ scattering amplitude. This form factor is taken in the form proposed by Ferrari and Selleri [24]:

$$
F\left(t_{1}\right)=\frac{1}{1+\left(m_{K}^{2}-t_{1}\right) / t_{0}} .
$$

We fix the cutoff parameter $t_{0}$ via a fit to data on the total reaction cross section for $\gamma p \rightarrow K^{+} K^{-} p$. The resulting value is $t_{0}=0.33 \mathrm{GeV}^{2}$, which corresponds to a cutoff mass of $\Lambda=0.76$ $\mathrm{GeV}$ within a standard monopole form factor. The $\gamma K^{+} K^{-}$ vertex contains no form factor because of the Ward identity. The amplitude for $t$-channel $K^{+}$exchange has the same structure except that then $T_{K^{+} p}$ enters and, of course, the form factor $F$ in the $K N \rightarrow K N$ vertex could be different as well. We implement current conservation by adopting the prescription of deForest and Walecka to replace the longitudinal current $\hat{q} \cdot \boldsymbol{j}(\boldsymbol{q})$ by $j^{0}(\boldsymbol{q}) q^{0} / q$ [25], where $q$ denotes the photon momentum. This corresponds to the addition of an appropriate contact interaction between the photon, kaon, and nucleon. Note, that such contact terms arise also in the formalism of Haberzettl [26] which was recently applied to the photoproduction of kaons in the vicinity of the pentaquark [27], though one must say that in the latter case this prescription is based on the Ward-Takahashi identity while it is essentially ad hoc in our case.

In case of kaon exchange the elementary $(\bar{K} N$ and $K N)$ scattering processes take place even farther off-shell than those in the reaction $\gamma p \rightarrow \pi^{+} \pi^{-} p$. Still we believe that it is a good working hypothesis to use the on-shell amplitudes in Eq. (1) but take into account the offshellness of the actual elementary reaction effectively by introducing a form factor. It rests on the 
expectation that the $s_{2}$ (and $t_{2}$ ) dependence of the elementary amplitudes, c.f. Eq. (1), remain largely unchanged when the reaction takes place off-shell. This expectation is supported by experience with other reactions. For example, one knows from meson-production reactions in $N N$ collisions $(p p \rightarrow p p x, x$ $\left.=\pi, \eta, \eta^{\prime} \ldots\right)$ that the energy dependence of the reaction cross section is strongly dominated by the energy $(s)$ dependence of elastic $p p$ scattering, even though in the production reaction the relevant $p p$ interaction in the final state takes place far off-shell, see, e.g., Ref. [28].

In the Drell formulation all possible resonances coupled to either the $K N$ or $\bar{K} N$ channels enter the calculations through the $K N \rightarrow K N$ or $\bar{K} N \rightarrow \bar{K} N$ scattering or charge exchange amplitudes. Keeping in mind that the available data on hyperonresonance properties are quite uncertain [5], calculations utilizing the experimentally available $\bar{K} N \rightarrow \bar{K} N$ amplitudes at least allow one to incorporate the best phenomenological information into the treatment of the reaction $\gamma N \rightarrow K \bar{K} N$. In that respect the Drell mechanism provides consistency between the data on $\bar{K} N \rightarrow \bar{K} N$ elastic scattering and $K \bar{K} N$ photoproduction. Similar considerations hold for the $K N$ system.

Originally Söding [13] proposed that one parameterizes the invariant elastic amplitude (in our case $T_{\bar{K} N}$ and $T_{K N}$ ) directly from the data on the total cross section $\sigma_{\text {tot }}$ by exploiting the optical theorem,

$$
T_{K N}\left(s_{2}, t_{2}\right)=-2 i q_{2} \sqrt{s_{2}} \sigma_{t o t}\left(s_{2}\right) \exp \left(b t_{2}\right),
$$

where $s_{2}$ is the $K N(\bar{K} N)$ invariant mass squared, $q_{2}$ is the modulus of the c.m. momentum and $t_{2}$ is the squared fourmomentum transferred from the initial to the final nucleon. Here $b$ is the exponential slope of the $t$ dependence taken from the data. This approximation is, in principle, sufficient to calculate the energy dependence of the reaction and the $K N(\bar{K} N)$ mass distribution. However, for an application involving cuts on certain momenta and angles one needs the specific dependence of the $K N(\bar{K} N)$ amplitude on those quantities and then the simple $\exp (b t)$ ansatz is not adequate.

In order to circumvent this problem Berestetsky and Pomeranchuk [29] and also Ferrari and Selleri [30] proposed that one parameterize the invariant scattering amplitude directly from differential elastic scattering cross section data:

$$
\left|T_{K N}\left(s_{2}, t_{2}\right)\right|^{2}=64 \pi s_{2} q_{2}^{2} \frac{d \sigma_{e l}\left(s_{2}\right)}{d t_{2}} .
$$

At least this approximation allows one to account for many details of the nuclear reactions and to reconstruct general features of the underlying dynamics [31-33]. However, even this form is not suitable for the present case where two different amplitudes connected with $K^{+}$and $K^{-}$meson exchanges, respectively, enter the calculation coherently.

Thus, the most consistent way is to use the partial wave decomposition of the scattering amplitude given by

$$
\begin{array}{r}
G\left(s_{2}, \theta\right)=\frac{1}{q_{2}} \sum_{l}\left[(l+1) T_{l}^{+}\left(s_{2}\right)+l T_{l}^{-}\left(s_{2}\right)\right] P_{l}(\cos \theta), \\
H\left(s_{2}, \theta\right)=\frac{\sin \theta}{q_{2}} \sum_{l}\left[T_{l}^{+}\left(s_{2}\right)-T_{l}^{-}\left(s_{2}\right)\right] \frac{d P_{l}(\cos \theta)}{d \cos \theta},
\end{array}
$$

where $G$ and $H$ are the spin-nonflip and spin-flip amplitudes [34], respectively, $P_{l}(\cos \theta)$ are the Legendre polynomials and $\theta$ is the scattering angle in the c.m. system. The partial wave amplitudes (for the $K N$ as well as the $\bar{K} N$ systems) are defined as

$$
T_{l}^{ \pm}=\frac{\eta_{l}^{ \pm} \exp \left(2 i \delta_{l}^{ \pm}\right)-1}{2 i}
$$

where $\eta_{l}^{ \pm}$and $\delta_{l}^{ \pm}$denote the inelasticity and the phase shift, respectively, for the total angular momentum $J=l \pm 1 / 2$. The relation between the invariant $G$ and $H$ scattering amplitudes and various scattering observables can be found, for example, in Ref. [34]. An application of the partial wave amplitudes to the analysis of the reaction $\gamma p \rightarrow \pi^{+} \pi^{-} p$ for the Drell mechanism contribution using the Söding model is described in detail in Ref. [15]. Note that the partial wave decomposition is given in the isospin basis and therefore any of the final channels of the reaction $\gamma N \rightarrow K \bar{K} N$ can be calculated.

In our investigation we use the $K N$ amplitudes from the Jülich meson-exchange model. A detailed description of the model is given in Refs. [35,36]. The model yields a satisfactory reproduction of the available experimental information on elastic and charge exchange $K N$ scattering including angular spectra and polarization data up to an invariant mass of the $K N$ system of $\sqrt{s_{2}} \simeq 1.8 \mathrm{GeV}$. For higher energies we adopt the phenomenological ansatz given by Eq. (3) utilizing experimental data compiled in Ref. [37]. Let us mention in this context that the $K N$ amplitude from the Jülich model was used by us recently for determining new limits for the $\Theta^{+}$pentaquark width from the data available for the $K^{+} d \rightarrow K^{0} p p$ reaction [38] and also for an analysis of the DIANA experiment [39], where the $\Theta^{+}$pentaquark was reportedly observed in $K^{+}$meson collisions with $X e$ nuclei [40]. Therefore, as an advantage, our approach offers the possibility for the self-consistent inclusion of the $\Theta^{+}$pentaquark via the $K N$ scattering amplitude, where the latter has already been developed and compared to other available data $[38,40]$.

The $\bar{K} N$ amplitudes are reconstructed from the result of a multichannel partial wave analysis (PWA) [41] available for $\bar{K} N$ scattering for invariant collision energies $1.48 \leq \sqrt{s_{2}} \leq 2.17$ $\mathrm{GeV}$. For $\sqrt{s_{2}} \leq 1.48 \mathrm{GeV}$ we adopt the $K$-matrix solution of Martin and Ross [42], which satisfactorily describes all available experimental results below the energy of $1.48 \mathrm{GeV}$. The $K$-matrix solution includes only $S$-waves for the $I=0$ and $I=1$ channels. However, the data shown in Ref. [42] illustrate that for $\sqrt{s} \leq 1.48 \mathrm{GeV}$ the contribution from higher partial waves is small.

The usefulness of the Drell approach with respect to analyzing the data becomes obvious by first looking at results for the cross section of the reactions $K^{-} p \rightarrow K^{-} p$ and $K^{-} p \rightarrow \bar{K}^{0} n$, shown in Fig. 2. Here the experimental information, taken from Refs. [5,43,44], is compared with calculations utilizing the total $\bar{K} N$ scattering amplitude of Ref. [41] (solid lines) with results that take into account only the contribution from the $D_{03}$ partial wave (dashed lines). (We use here the standard nomenclature $L_{I 2 J}$.) Note that the $D_{03}$ partial wave contains the $\left(I\left(J^{P}\right)=0\left(\frac{3}{2}^{-}\right)\right) \Lambda(1520)$ resonance with nominal mass $1519.5 \pm 1.0 \mathrm{MeV}$ and full width $15.6 \pm 1.0 \mathrm{MeV}$ [5]. While the $K^{-} p \rightarrow K^{-} p$ reaction shows almost no trace of the $\Lambda(1520)$, 

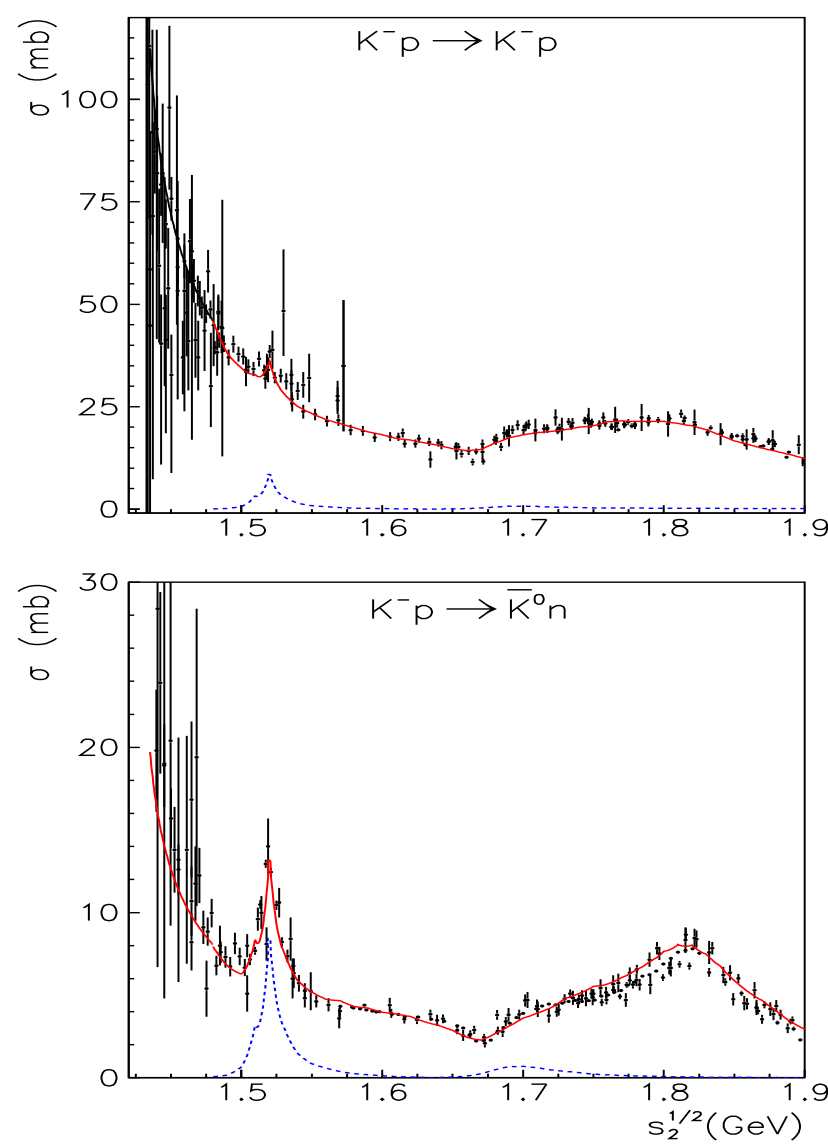

Fig. 2. The $K^{-} p \rightarrow K^{-} p$ and $K^{-} p \rightarrow \bar{K}^{0} n$ cross sections as a function of the invariant collision energy. The solid lines show results based on the full reaction amplitude, while the dashed lines indicate contribution from the $D_{03}$ partial wave alone. The data are taken from Refs. [5, 43,44].

the charge-exchange reaction $K^{-} p \rightarrow \bar{K}^{0} n$ clearly indicates a resonance structure. This difference can be understood by recalling that the reaction amplitude for the $K^{-} p \rightarrow K^{-} p$ channel consists of (half of) the sum of the $I=0$ and $I=1$ amplitudes, while the $K^{-} p \rightarrow \bar{K}^{0} n$ reaction is given by (half of) their difference. Since both $I=0$ and $I=1$ amplitudes contain large nonresonant contributions, it turns out that this nonresonant background cancels to a large extent for the $K^{-} p \rightarrow \bar{K}^{0} n$ amplitude. Fig. 2 demonstrates quite strikingly that the contribution of the $D_{03}$ partial wave to the $K^{-} p \rightarrow K^{-} p$ reaction is almost negligible compared with the nonresonant background, while its contribution to the charge-exchange channel is sizeable.

This observation suggests that kaon exchange (viz. the Drell mechanism) should not produce a pronounced $\Lambda(1520)$ signal in the invariant mass of the $K^{-} p$ system for the reaction $\gamma p \rightarrow K^{+} K^{-} p$ but only in the $\gamma p \rightarrow K^{+} \bar{K}^{0} n$ channel. Consequently, if the reaction $\gamma p \rightarrow K^{+} K^{-} p$ does indeed show a substantial effect of the $\Lambda(1520)$ resonance, it is a strong indication that mechanisms other than the Drell mechanism dominate the reaction [6].

Fig. 2 illustrates also that any model calculation of the reaction $\gamma N \rightarrow K \bar{K} N$ where the employed $\bar{K} N \rightarrow \bar{K} N$ amplitude is constructed from hyperon resonances alone (cf. diagram c) in Fig. 1) - as it is the case in so-called resonance models - is definitely not realistic, since there are significant nonresonant contributions to the scattering amplitude. In this context let us emphasize that the $\bar{K} N \rightarrow \bar{K} N$ amplitude contains large contributions from higher partial waves. Fig. 3 shows the angular distribution of the $K^{-}$mesons in the c.m. system of the reaction $K^{-} p \rightarrow K^{-} p$ reaction, which clearly manifests the increasing importance of higher partial waves with increasing invariant collision energy, $\sqrt{s_{2}}$.

With the partial-wave decomposition for the amplitudes $G$ and $H$ given in Eq. (5) the differential cross section can be readily evaluated for the specific $D_{03}$ partial wave where the $\Lambda(1520)$ resonance occurs:

$$
\frac{d \sigma}{d \cos \theta}=|G|^{2}+|H|^{2}=\left|\frac{T_{2}^{-}\left(s_{2}\right)}{q_{2}}\right|^{2}\left(3 \cos ^{2} \theta+1\right) .
$$

The resulting angular dependence, $1+3 \cos ^{2} \theta$, is shown by the dashed lines in Fig. 3, arbitrarily normalized to the data. It is evident that the $1+3 \cos ^{2} \theta$ function alone does not describe the data at $\sqrt{s}=1520 \mathrm{MeV}$; additional contributions, from other partial waves, are required. The solid lines in Fig. 3 represent results with the total reaction amplitude, i.e. with all partial waves including the $D_{03}$. It is interesting to note that while the $\Lambda(1520)$ resonance remains almost undetectable in the $K^{-} p \rightarrow$ $K^{-} p$ reaction cross section shown in Fig. 2, because of the large background, this resonance can be well reconstructed from an analysis of the angular distributions. A detailed discussion
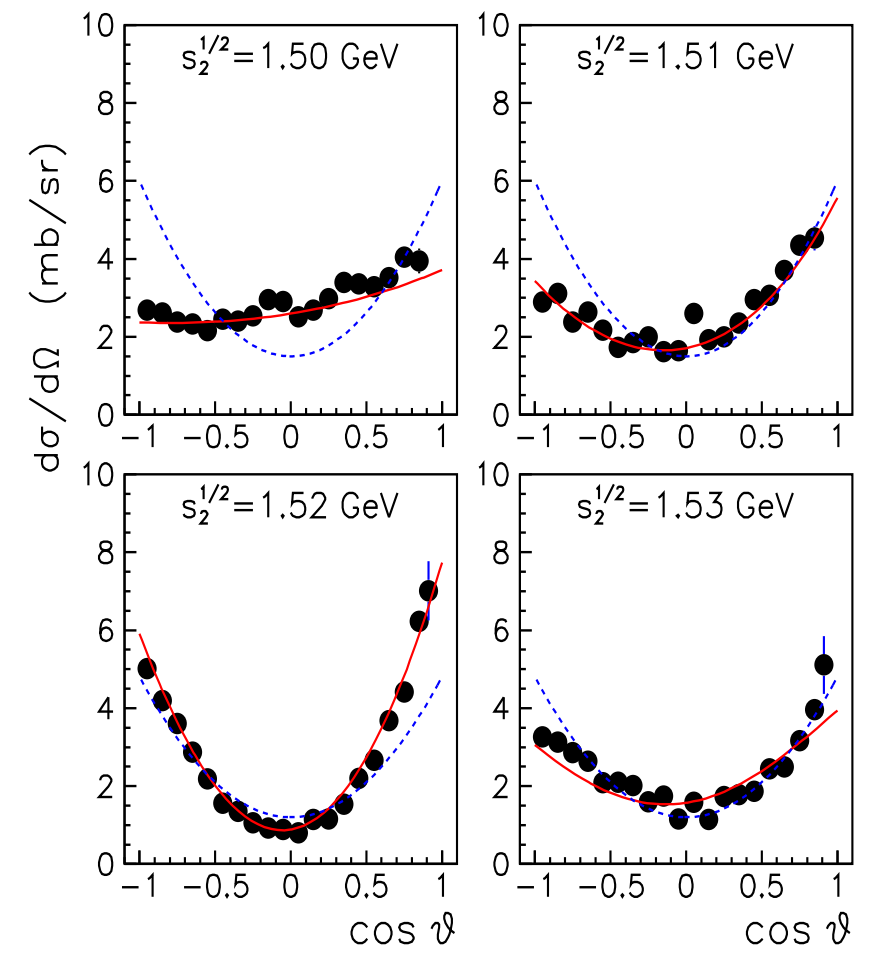

Fig. 3. Differential cross section for $K^{-} p$ scattering as a function of the $K^{-}$meson scattering angle in the c.m. system for different invariant collision energies, $\sqrt{s_{2}}$. The solid lines show results based on the full reaction amplitude, while the dashed lines indicate the distribution given by $1+3 \cos ^{2} \theta$ normalized to the data. The data are taken from Refs. [43,44]. 


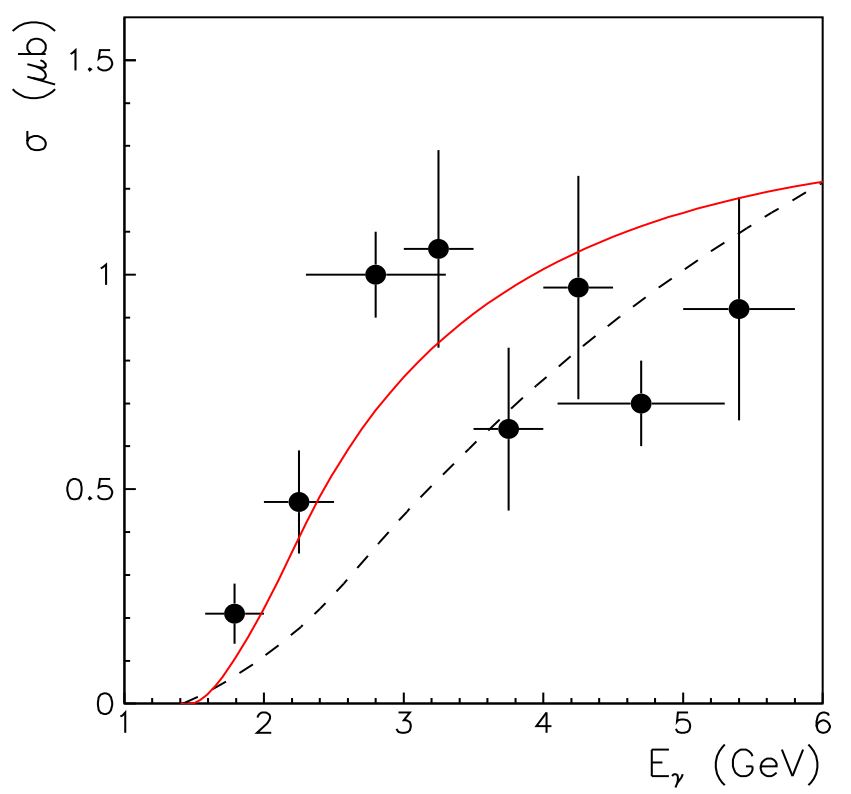

Fig. 4. Cross section of the reaction $\gamma p \rightarrow K^{+} K^{-} p$ as a function of the photon energy. The dashed line is the result obtained with a constant reaction amplitude while the solid line shows the result for the Drell mechanism. The symbols represent data collected in Ref. [46].

of the $K^{-}$meson angular spectra at energies around the $\Lambda(1520)$ resonance is given in Ref. [45].

Fig. 4 shows the cross section of the $\gamma p \rightarrow K^{+} K^{-} p$ reaction as a function of photon energy. The circles represent experimental information collected in Ref. [46].

The total $\gamma p \rightarrow K^{+} K^{-} p$ cross section is given by integration of the Chew-Low distribution [47]

$$
\frac{d \sigma}{d s_{2} d t_{1}}=\frac{\left|\mathcal{M}_{K^{+}}+\mathcal{M}_{K^{-}}\right|^{2}}{2^{9} \pi^{3}\left(s-m_{N}^{2}\right)^{2}} \frac{\lambda^{1 / 2}\left(s_{2}, m_{K}^{2}, m_{N}^{2}\right)}{s_{2}},
$$

where the function $\lambda$ is defined by

$$
\lambda(x, y, z)=\frac{(x-y-z)^{2}-4 y z}{4 x} .
$$

The dotted line in Fig. 4 is the result with a constant invariant $\gamma p \rightarrow K^{+} K^{-} p$ reaction amplitude, i.e. $\mathcal{M}=$ const., suitably adjusted to the data The full calculation (solid line in Fig. 4) reproduces the energy dependence of the data reasonably well from threshold up to a photon energy of $\simeq 5 \mathrm{GeV}$-i.e., over the whole energy range covered by the LAMP2 experiment. Also in this case the absolute value of the model result was adjusted to the data, namely by tuning the cutoff mass in the form factor Eq. (2), cf. above. Indeed, the experimental $K N$ invariant mass spectra which we want to investigate with our model are given without absolute normalization, so that the overall normalization is irrelevant for our application anyway.

\section{Invariant $K N$ mass spectra from the reaction $\gamma p \rightarrow K^{+} K^{-} p$}

The $K^{+} p$ and $K^{-} p$ invariant mass spectra were measured by the LAMP2 Group [6] in the reaction $\gamma p \rightarrow K^{+} K^{-} p$ with a tagged photon beam with an energy $2.8<E_{\gamma}<4.8 \mathrm{GeV}$. Figs. 5,6 show the invariant mass spectra for the $K^{+} p$ and $K^{-} p$ subsystems. Note that for the photon energy of $4.8 \mathrm{GeV}$ the maximal invariant mass of the $K N$ system ranges up to roughly $2.65 \mathrm{GeV}$. The experimental $K^{-} p$ mass spectrum is provided only up to $\simeq 1.65 \mathrm{GeV}$ in Ref. [6], while the data for the $K^{+} p$ mass distribution are given over almost the whole available range.

The dotted lines in Figs. 5,6 represent the phase space distribution

$$
\frac{d \sigma}{d s_{2}}=\frac{\lambda^{1 / 2}\left(s, s_{2}, m_{K}^{2}\right) \lambda^{1 / 2}\left(s_{2}, m_{N}^{2}, m_{K}^{2}\right)}{2^{8} \pi^{3} s s_{2}\left(s-m_{N}^{2}\right)}|\mathcal{M}|^{2},
$$

evaluated for the fixed averaged photon energy of $3.8 \mathrm{GeV}$ and assuming a constant reaction amplitude, i.e. $\mathcal{M}=$ const. In our notation the invariant $K N$ mass $M(K N)$ is given by $M(K N)=\sqrt{s_{2}}$. Since the experimental results are available only with arbitrary normalization we adjust the amplitude $\mathcal{M}$ to the $K^{-} p$ background at invariant masses above the resonance structure corresponding to the $\Lambda(1520)$, i.e. around $1570 \mathrm{MeV}$. The same normalization factor is then used also in the evaluation of the $K^{+} p$ mass spectrum.

From Fig. 5 it is obvious that the phase space distribution differs from the measured mass dependence of the $K^{+} p$ spectrum. On the other hand the measurement exhibits large fluctuations and therefore it is hard to say to what extent the structure of the data reflects underlying physics or whether it is simply a consequence of poor statistics. Nonetheless, it appears that there is an excess of events at high $K^{+} p$ invariant masses. Note

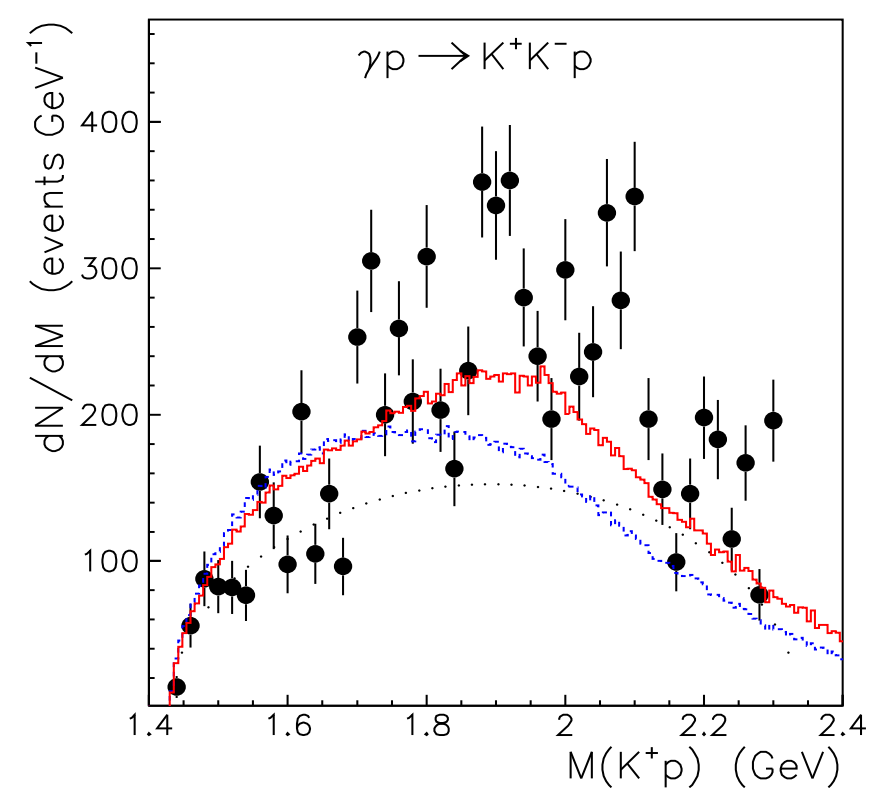

Fig. 5. The $K^{+} p$ invariant mass spectrum from the $\gamma p \rightarrow K^{+} K^{-} p$ reaction. The dotted line is the result obtained with a constant reaction amplitude for the fixed photon energy $3.8 \mathrm{GeV}$. The dashed line shows the result for the Drell mechanism, while the solid line is obtained after inclusion of additional $K^{*}$ meson exchange for $\Lambda(1520)$ production. Here the photon energy is averaged over $2.8<E_{\gamma}<4.8 \mathrm{GeV}$ in consistency with the experiment. The data are taken from Ref. [6]. 


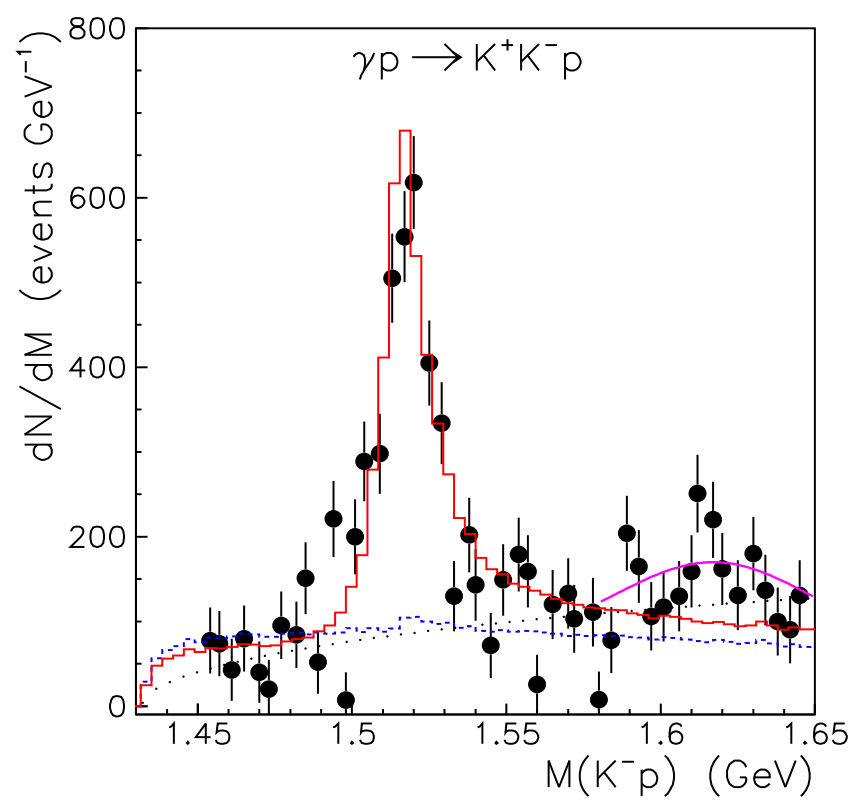

Fig. 6. The $K^{-} p$ invariant mass spectrum for the reaction $\gamma p \rightarrow K^{+} K^{-} p$. Same description of the curves as in Fig. 5. The thin solid line indicates the contribution of a $\Lambda(1600) P_{01}$ resonance, discussed in Sect. 4. The circles are data taken from Ref. [6].

that the incident photon energy distribution in the experiment is proportional to $E_{\gamma}^{-1}$ and, therefore, naively one expects more events at low $K^{+} p$ masses. Fortunately, the $K^{-} p$ data themselves indicate a possible explanation of that problem, namely the presence of a clean resonance structure around $M\left(K^{-} p\right)$ $=1520 \mathrm{MeV}$ and probably at around $M\left(K^{-} p\right)=1620 \mathrm{MeV}$, cf. Fig. 6. One would expect that a large contribution at low $K^{-} p$ masses, like that associated with the $\Lambda(1520)$ resonance, would be kinematically reflected in the $K^{+} p$ mass spectrum and cause a substantial shift to higher $M\left(K^{+} p\right)$ values in the latter.

Within an analysis based on the Drell mechanism the resonances at $M\left(K^{-} p\right)=1520 \mathrm{MeV}$ and $M\left(K^{-} p\right)=1620 \mathrm{MeV}$ enter via the elastic $K^{-} p \rightarrow K^{-} p$ scattering amplitude. Indeed the partial wave analysis includes the $\Lambda(1520) J^{P}=\frac{3}{2}^{-}$and A(1600) $J^{P}=\frac{1}{2}^{+}$resonances in the $D_{03}$ and the $P_{01}$ partial waves, respectively. However, the elastic scattering data depicted in Fig. 2 as well as the $K^{-} p$ amplitude of the PWA do not show any obvious signal of those resonances, as already pointed out earlier. Therefore, we do not expect that a calculation based on the Drell mechanism will be able to reproduce these resonances as observed in the $K^{-} p$ invariant mass spectrum of the reaction $\gamma p \rightarrow K^{+} K^{-} p$.

This is indeed the case as can be seen from our results for the Drell diagrams which correspond to the dashed histograms in Figs. 5 and 6. Obviously, there is only a small, hardly noticeable enhancement in the $K^{-} p$ mass spectrum due to the $\Lambda(1520)$ resonance and the same is the case also for the $\Lambda(1600)$ resonance. The $E_{\gamma}^{-1}$ factor of the photon energy distribution is now included in the calculation and it is obvious from Fig. 5 how the spectrum is shifted to lower $K^{+} p$ invariant masses because of that. Note that the results were normalized in such a way that we are in line with the low invariant-mass spec- trum of the $K^{-} p$ system and we use again the same normalization for the $K^{-} p$ and $K^{+} p$ results. Also, our calculations were developed in line with the Söding model [13] in order to allow for possible kinematic cuts and simulation of the detector acceptance of current experiments. The integration over phase space is based on the Monte-Carlo method that allows one to construct an event generator. That is why the calculations are shown as histograms.

The results in Fig. 6 make it clear that additional reaction mechanisms need to be considered if one wants to describe the experimental $K^{-} p$ invariant mass spectrum. To account for such additional mechanisms we resort here to $K^{*}$ meson exchange with subsequent excitation of the $\Lambda(1520)$ resonance, as depicted by the diagram d) in Fig. 1. Indeed, the experimental helicity-frame angular distribution of the $4(1520)$ decay for the reaction $\gamma p \rightarrow K^{+} K^{-} p$ [6] strongly suggests that $K^{*}$ exchange should play an important role. Note, however, that while our calculation of the Drell mechanism is considerably constrained by experimental information on the $K N$ and $\bar{K} N$ amplitude and therefore can be considered as a solid background for the reaction $\gamma N \rightarrow K \bar{K} N$, this is not the case for the $K^{*}$ exchange because there are no data on the reaction $K^{*} N \rightarrow K N$ (or $\left.\overline{K^{*}} N \rightarrow \bar{K} N\right)$. Furthermore, it is possible that many different $\Lambda$ and $\Sigma$ hyperon resonances are excited in the $\gamma N \rightarrow K \bar{K} N$ reaction through $K^{\star}$ meson exchange. But the relevant coupling constants of the $K^{*}$ meson to the hyperon resonances are completely unknown. Thus, the best one can do is to start with the general structure of the $K^{\star}$ exchange amplitude for hyperon resonance photoproduction and to fit the unknown parameters to the presently available data. For reasons of simplicity we decided to take into account only the excitation of the $\Lambda(1520)$ resonance. This has the advantage that one can at least use the available data on the reaction $\gamma p \rightarrow K^{+} \Lambda(1520)$ to constrain the parameters. Details about the structure of the employed $K^{\star}$ exchange amplitude are given in the Appendix, together with a comparison to the $\gamma p \rightarrow K^{+} \Lambda$ (1520) data.

We should also mention that, of course, it is only an assumption that the needed additional contributions come from $K^{\star}$ exchange alone. In principle, any other meson exchange could contribute as well. And even scenarios like those considered in Refs. [20,21], where the bulk of the $\gamma p \rightarrow K^{+} \Lambda(1520)$ cross section is generated by contact terms, are possible.

The solid histograms in Figs. 5 and 6 represent the $K^{+} p$ and $K^{-} p$ invariant mass spectra evaluated with $K$ and $K^{*}$ meson exchanges. The calculation reproduces quite well the experimental $K^{-} p$ invariant mass spectra. Note that we do not include the $\Lambda(1600)$ resonance excitation via $K^{*}$ meson exchange since there is no experimental information about the energy and $t$-dependence of its production and also no data on the spin density matrix. As a consequence our calculation underestimates the $K^{-} p$ spectrum in the corresponding invariant mass region. However, we will come back to this issue in the next section.

The inclusion of $K^{*}$ meson exchange also improves the description of the $K^{+} p$ invariant mass spectrum. In particular, we now find a noticeable enhancement at higher invariant masses. However, we still underestimate the $K^{+} p$ mass spectrum at masses above $1.8 \mathrm{GeV}$. The discrepancy might be related to the production of other hyperon resonances with masses above 
$1.650 \mathrm{GeV}$, i.e. in the $K^{-} p$ invariant mass region not covered by the LAMP2 experiment [6]. If such resonances can be produced by $K^{*}$ meson exchange they would be seen in the $K^{-} p$ invariant mass distribution - and adding their contributions to the $\gamma p \rightarrow K^{+} K^{-} p$ amplitude might improve the description of the $K^{+} p$ mass spectrum. However, at this stage and without data, this remains pure speculation.

\section{Application to the SAPHIR data}

As a first application of our model and in order to demonstrate its potential for future analyses we present here a comparison with data on the reaction $\gamma N \rightarrow K \bar{K} N$ taken recently by the SAPHIR collaboration at ELSA (Bonn). Their study of the channel $\gamma p \rightarrow K^{+} K_{s}^{0} n$ was among the first which revealed evidence for the $\Theta^{+}(1540)$ pentaquark [3]. However, the group has also provided invariant mass spectra for the $K^{-} p$ and $K^{+} p$ systems from the reaction $\gamma p \rightarrow K^{+} K^{-} p[3,48]$ which we want to analyze now. In their experiment the photon energy range is $1.74 \leq E_{\gamma} \leq 2.6 \mathrm{GeV}$, i.e. significantly lower than in the LAMP2 experiment.

The results of our model (including both the Drell mechanism and the $K^{*}$ exchange contribution) are shown in Fig. 7 for the SAPHIR experiment. Since the data are without absolute normalization we readjusted our model predictions to the maximum of the $K^{+} p$ invariant mass spectrum. It is obvious from Fig. 7 that the $K^{+} p$ mass spectrum is nicely reproduced over the whole invariant-mass range. Moreover, at the same time (and with the same normalization) there is also excellent agreement with the $K^{-} p$ spectrum in the region of the $\Lambda(1520)$ resonance, as well as at high invariant masses, cf. Fig. 7. However, there is some excess in the experimental mass spectrum around $1600 \mathrm{MeV}$. If one subtracts the model prediction from the SAPHIR data one obtains the results shown in Fig. 8. It is interesting to see that this difference strongly resembles a resonance signal, something one would not have guessed easily from the original data in Fig. 7. Fitting this difference with a relativistic Breit-Wigner amplitude Eq. (19) yields the values

$$
M_{R}=1617 \pm 2 \mathrm{MeV}, \quad \Gamma_{R}=117 \pm 4 \mathrm{MeV},
$$

with a $\chi^{2}$ per data point of $\chi^{2} / N=4$. The large $\chi^{2} / N$ reflects the considerable fluctuation of the data.

We are inclined to identify this structure with the $\Lambda(1600)$ $P_{01}$ resonance [5], though we are aware that further and more careful analyses are needed in order to substantiate such a claim, specifically because the PDG lists also other hyperon resonances in this energy region [5], e.g. the $\Sigma(1580)$ and $\Sigma(1620)$. Independently of that, we believe that this particular case already demonstrates very clearly the power of a reliable model for the background to the reaction $\gamma N \rightarrow K \bar{K} N$ in the analysis of experimental data. Two further short comments are in order: First, the SAPHIR collaboration is in the process of analyzing the $4(1520)$ signal in their data [49] and, second, we speculate that the sharp signal on the left side of Fig. 8 is related to the excitation of the $\Lambda(1405)$ through $K^{*}$ exchange. This deserves further study.

As a consistency check, we have added the contribution of the $\Lambda(1600)$ resonance to the $K^{-} p$ invariant mass spectrum of
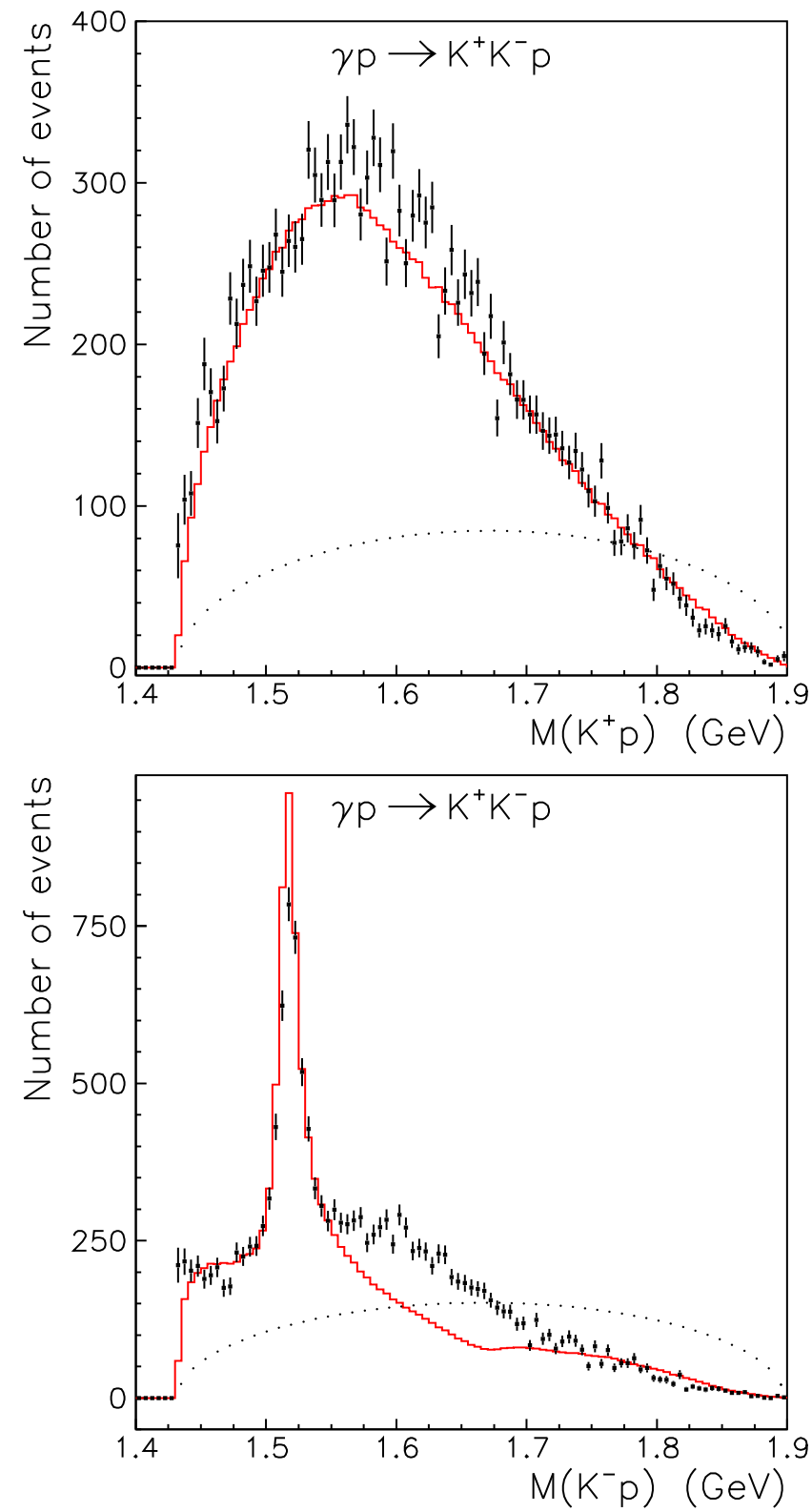

Fig. 7. The $K^{+} p$ and $K^{-} p$ spectra for the $\gamma p \rightarrow K^{+} K^{-} p$ reaction measured by SAPHIR Collaboration $[3,48]$. Same description of curves as in Fig. 6.

Ref. [6], as shown by the thin solid line in Fig. 6. One can see that its contribution is indeed consistent with these data, however, the figure also underlines our earlier statement that the data from [6] are not sufficiently precise to allow an extraction of resonance properties for invariant masses above the $\Lambda(1520)$.

Coming back to the $K^{+} p$ mass spectrum of the SAPHIR collaboration we would like to emphasize that its dependence on the invariant mass $M\left(K^{+} p\right)$ is rather smooth up to the highest values as can be seen in Fig. 7. Therefore, we believe that the structures seen in the corresponding LAMP2 data, cf. Fig. 5, are most likely fluctuations associated with low statistics. This concerns presumably also events at those higher invariant masses which are not covered by the SAPHIR data. Anyway, it would 


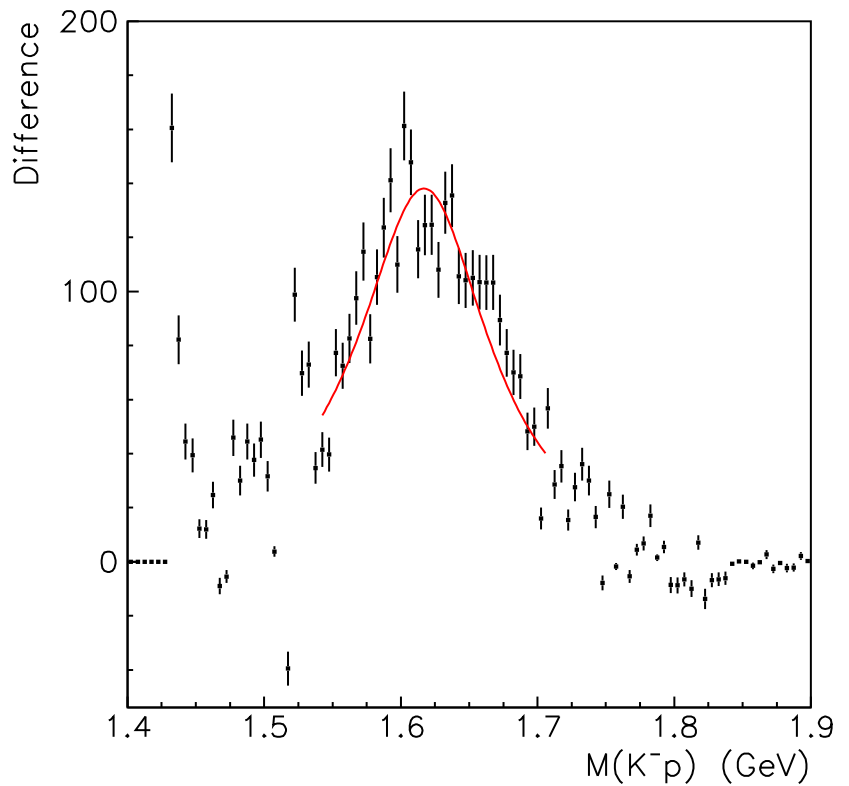

Fig. 8. The difference between the SAPHIR data $[3,48]$ for the $K^{-} p$ invariant mass spectra of $\gamma p \rightarrow K^{+} K^{-} p$ and our model prediction. The solid line indicates the fit with a relativistic Breit-Wigner function.

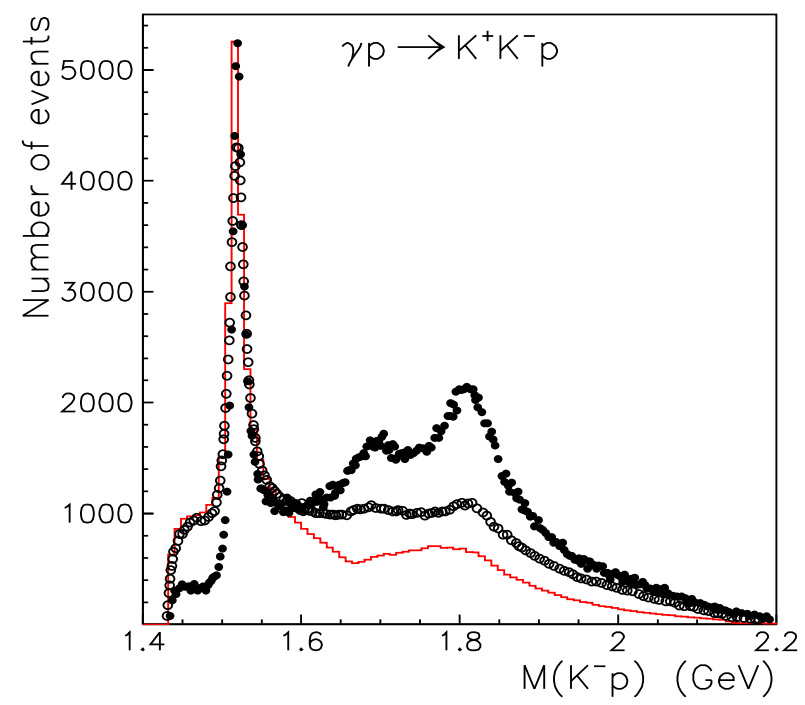

Fig. 9. The $K^{-} p$ spectrum for the reaction $\gamma p \rightarrow K^{+} K^{-} p$ as measured by the CLAS Collaboration [50]. The open and closed circles show results obtained with different criteria for the final-state particle reconstruction. The data are not efficiency corrected. The solid histogram is our calculation for the photon energies $1.8 \leq E_{\gamma} \leq 3.8 \mathrm{GeV}$.

be interesting to explore this region again experimentally with much better statistics than what was available in the LAMP2 experiment.

\section{Application to the CLAS data}

Recently also the CLAS Collaboration reported [50] new results on the reaction $\gamma p \rightarrow K^{+} K^{-} p$ for photon energies of $1.8 \leq$ $E_{\gamma} \leq 3.8 \mathrm{GeV}$. However, unfortunately their data are not efficiency corrected [51]. Thus a direct comparision of our results with those data and specifically, an analysis similar to the one for the SAPHIR data with the aim to extract possible contributions from hyperon resonances is not possible at this stage. Still we find it interesting to compare the prediction for the $K^{-} p$ invariant mass spectrum resulting from the Drell mechanism with those data.

The solid histogram in Fig. 9 shows the result of our calculation based on the diagrams a) and b) of Fig. 1 and taking into account additional contributions to the $\Lambda(1520)$ production due to $K^{*}$-exchange. The uncorrected experimental spectra are from Ref. [50], where the two sets of data points (open and closed squares) were obtained by using different criteria for the final-state particle reconstruction. For the comparision we normalized both sets of the data and our calculation at the maximal yield of the $\Lambda(1520)$ resonance. Our calculation clearly indicates that the $K^{-} p$ mass spectra at $1.8 \leq E_{\gamma} \leq 3.8 \mathrm{GeV}$ provide an excellent tool to identify possible additional contributions from high-mass hyperon resonances. Also, an additional measurement of the angular spectra in the Gottfried-Jackson system at fixed $K^{-} p$ masses would provide valuable information for the hyperon resonances spectroscopy. Thus, it will be interesting to analyze the CLAS data within our model once these data are efficiency corrected. Only then concrete conclusion can be made.

\section{Conclusion}

We have studied the reaction $\gamma N \rightarrow K \bar{K} N$ utilizing the Drell mechanism and taking into account the full reaction amplitudes for the $K N$ and $\bar{K} N$ subsystems. Our results show that the Drell mechanism for kaon exchange alone is not sufficient to describe the available data on the $K^{+} p$ and $K^{-} p$ invariant mass spectra. Thus, we have included $K^{*}$ meson exchange as an additional reaction mechanism. By assuming a large $K^{*}$ coupling to the $\Lambda(1520)$ resonance a quantitative description of the $K^{-} p$ invariant mass spectrum can be achieved. Moreover, our model calculation also yields a good overall reproduction of the cross section data for the photoproduction of the $\Lambda(1520)$ resonance, i.e of its energy and $t$-dependence.

In the paper by Barber et al. [6] it was argued, based on the measured distribution of the $\Lambda(1520)$ decay into the $K^{-} p$ channel in the Gottfried-Jackson frame, that kaon exchange alone cannot account for the $\Lambda(1520)$ production mechanism. Our investigation, utilizing the Drell mechanism and experimental information on the $K N$ and $\bar{K} N$ scattering amplitudes, can be considered as an independent confirmation of this conclusion.

The $\Lambda(1520)$ excitation in photoproduction illustrates an aspect that is also relevant for discussions concerning the $\Theta^{+}$ pentaquark. While the $\Lambda(1520)$ resonance remains practically undetectable in $K^{-} p$ elastic scattering (see Fig. 2), the resonance is clearly visible in the photoproduction reaction shown in Fig. 6. Evidently, the difference between elastic scattering 
and photoproduction must involve reaction mechanisms or channels which are not accessible in elastic scattering - such as the $K^{*}$ meson exchange which we assumed in the present study. The situation for $\Theta^{+}(1540)$ production might be similar. Different reaction mechanisms/channels could be quite selective to the $\Theta^{+}$excitation and, consequently, could be a natural reason why the pentaquark was observed in some experiments but not in others. The $4(1520)$ photoproduction is an excellent example of such a situation. Clearly, at present, in either case the reaction mechanisms governing the photoproduction are not yet identified and, moreover, there are no reasons to believe that they might be similar, let alone the same.

As a first application, we have analyzed the SAPHIR data on $\gamma p \rightarrow p K^{+} K^{-}$and shown that our approach gives an excellent description of the $K^{+} p$ mass distribution for all energies and the $K^{-} p$ mass distribution in the vicinity of the $\Lambda(1520)$ resonance and for energies above $1.7 \mathrm{GeV}$. We have shown that the remaining strength can be well described by a resonance with the parameters given in Eq. (11) - this state could be the $\Lambda(1600) P_{01}$ resonance of the PDG listing.

Since our model allows us to study not only the invariant mass spectra but also momentum and angular distributions of the final particles, it is also possible to compare the model results with more exclusive (i.e. differential) data and to investigate the possible role of the detector acceptance and kinematical cuts in the extraction of specific resonances, like the $\Theta^{+}(1540)$ pentaquark, from present or future experiments [52].

\section{Acknowledgments}

We would like to thank D. Diakonov, K. Hicks, N.N. Nikolaev, W. Schäfer and S. Stepanyan for useful discussions. We are grateful to J. Barth, F. Klein, M. Ostrick and W. Schwille for supplying us with the SAPHIR data and for comments. This work was partially supported by the Department of Energy under contract DE-AC05-84ER40150 under which SURA operates Jefferson Lab and by Deutsche Forschungsgemeinschaft through funds provided to the SFB/TR 16 "Subnuclear Structure of Matter". This research is part of the EU Integrated Infrastructure Initiative Hadron Physics Project under contract number RII3-CT-2004-506078. A.S. acknowledges support by the COSY FFE grant No. 41445400 (COSY-067).

\section{A The $K^{*}$ meson exchange contribution}

In this appendix we specify the $K^{*}$ meson exchange contribution to the reaction $\gamma p \rightarrow K^{+} K^{-} p$ used in our model calculation. Since the energy range where the experimental information is available, $2.8 \leq E_{\gamma} \leq 4.8 \mathrm{GeV}$, is already too high for employing effective Lagrangians [23], we resort to Regge phenomenology. The reaction mechanism is depicted in Fig. 1d), where we take into account only the excitation of the $\Lambda(1520)$ resonance. This has the advantage that we can use available data on the reaction $\gamma p \rightarrow K^{+} \Lambda(1520)$ to constrain the free parameters. Clearly, other hyperon resonances could contribute as well and there should be also a nonresonant background for $K^{*}$ exchange. However, it is impossible to constrain those contributions from data and therefore we leave them out altogether.
Let us first specify the amplitude for the elementary reaction $\gamma p \rightarrow K^{+} \Lambda(1520)$. We use phenomenological helicity amplitudes for the single $t$-channel meson exchange Regge pole for the process $a b \rightarrow 12$,

$$
M_{\lambda_{2} \lambda_{b}}^{\lambda_{1} \lambda_{a}}\left(s, t_{1}\right)=-V_{x}^{\lambda_{1} \lambda_{a}}\left(t_{1}\right) R_{x}\left(s, t_{1}\right) V_{\lambda_{2} \lambda_{b}}^{x}\left(t_{1}\right) .
$$

Here $R_{x}$ is the Regge propagator for the exchange of the meson $x$,

$$
R_{x}\left(s, t_{1}\right)=\frac{1+(-1)^{s_{x}} \exp \left[-i \pi \alpha_{x}\left(t_{1}\right)\right]}{2 \sin \left[\pi \alpha_{x}\left(t_{1}\right)\right] \Gamma\left[l_{x}-\alpha\left(t_{1}\right)\right]}\left[\alpha^{\prime} s\right]^{\alpha_{x}\left(t_{1}\right)},
$$

where $s_{x}$ and $l_{x}$ are the spin of the exchanged meson and the spin of the lowest state of the Regge trajectory, respectively. For $K^{*}$ meson exchange we use $s_{K^{*}}=1, l_{K^{*}}=1$. The Regge trajectory $\alpha_{K^{*}}$ is taken as

$$
\alpha_{K^{*}}=1+\alpha^{\prime}\left(t-m_{K^{*}}^{2}\right),
$$

with $\alpha^{\prime}=0.9 \mathrm{GeV}^{-2}$ and $m_{K^{*}}=892 \mathrm{MeV}$. The vertex function $V$ is parameterized as

$$
V_{x}^{\lambda_{1} \lambda_{a}}\left(t_{1}\right)=\beta_{x}^{\lambda_{1} \lambda_{a}}\left[t_{1}-t_{m i n}\right]^{\left|\lambda_{1}-\lambda_{a}\right| / 2},
$$

where $\beta_{x}$ is a helicity coupling constant and $t_{\min }$ is the minimal four-momentum transfer. The last term in Eq. (15) ensures that the spin flip amplitude vanishes at forward direction. In principle each vertex should be dressed with a form factor, which can be determined from the $t$-dependence of the experimental data. When the masses of the initial and final particles are different, i.e. $m_{1} \neq m_{a}$ and $m_{2} \neq m_{b}$ then

$$
t_{\min } \simeq-\frac{\left(m_{a}^{2}-m_{1}^{2}\right)\left(m_{b}^{2}-m_{2}^{2}\right)}{s} .
$$

Finally, the differential cross section for the reaction $a b \rightarrow 12$ is given as

$$
\frac{d \sigma}{d t}=\frac{1}{\left(2 s_{a}+1\right)\left(2 s_{b}+1\right) 64 \pi \lambda\left(s, m_{a}^{2}, m_{b}^{2}\right)} \sum_{\lambda_{i}}\left|M_{\lambda_{2} \lambda_{b}}^{\lambda_{1} \lambda_{a}}\right|^{2},
$$

where $s_{a}$ and $s_{b}$ are the spins of the initial particles and the summation is over all helicity amplitudes. Within this approach it is straightforward to include any meson exchange and any initial and final states. A detailed comparison between model calculations and data from pion and kaon induced reactions are given in Refs. [53-56].

The principal uncertainties of this model are due to the unknown helicity couplings and possible contributions from different exchange trajectories. The model parameters can be fixed only by comparison with available experimental results. For instance, the energy dependence of the reaction cross section is driven by the energy dependence of the Regge propagator, which can be used for fixing the exchange trajectories. The $\gamma p \rightarrow K^{+} \Lambda(1520)$ reaction cross section was measured [6, 7] and is shown in Fig. 10 as a function of the photon energy. The dotted line shows the cross section for $M_{\lambda_{2} \lambda_{b}}^{\lambda_{1} \lambda_{a}}=$ const.

For $K^{*}$ exchange the $K^{*} K \gamma$ coupling constant can be determined from the $K^{*} \rightarrow K \gamma$ decay, but the couplings and possible form factor at the $K^{*} N \Lambda(1520)$ vertex are unknown. These 
uncertainties allow enough freedom to reproduce the absolute normalization of the data. Also, the energy dependence of the reaction cross section, and the $t$-dependence of the differential spectra and density matrix elements can be fitted simultaneously resulting in a quite reasonable determination of the free parameters.

The dashed line in Fig. 10 shows the result from $K^{*}$ meson exchange. It is in line with the data over the photon energy range covered by the LAMP2 experiment. In this particular fit to the data we omitted form factors at the vertices but adjusted instead the overall normalization of the total amplitude. Let us mention though, that in principle the coupling constants for each helicity amplitude should be different [53].

Additional information on the reaction mechanism is contained in the $t$-dependence. Note that the vertex functions of the helicity amplitudes given by Eq. (15) are proportional to $(-t)^{n / 2}$ with $n=\left|\lambda_{1}-\lambda_{a}\right|+\left|\lambda_{2}-\lambda_{b}\right|$ being the net helicity flip. This implies that the spin flip amplitudes vanish at forward direction and increase with $|t|$. Data on the differential cross section for $\gamma p \rightarrow K^{+} \Lambda(1520)$ as a function of $t$, the square of the four-momentum transferred, for photon energies $2.8<E_{\gamma}<$ $4.8 \mathrm{GeV} \mathrm{[6]} \mathrm{are} \mathrm{presented} \mathrm{in} \mathrm{Fig.} \mathrm{11.} \mathrm{The} \mathrm{dashed} \mathrm{line} \mathrm{is} \mathrm{our}$ result without form factors. Apparently it is in disagreement with the data. Indeed the calculated differential cross section increases with $|t|$, which is an unphysical dependence exhibited by the low $t$ Regge model. Thus, to describe the data one needs to introduce form factors. We take those form factors to be $F(t)=\exp (3 \mathrm{t})$ for all helicity amplitudes. Furthermore we assume that the form factors do not depend on the photon energy. This assumption is not necessarily correct because other higher-mass $e$ meson exchanges might contribute and could be dressed with different form factors. Alternatively one can in-

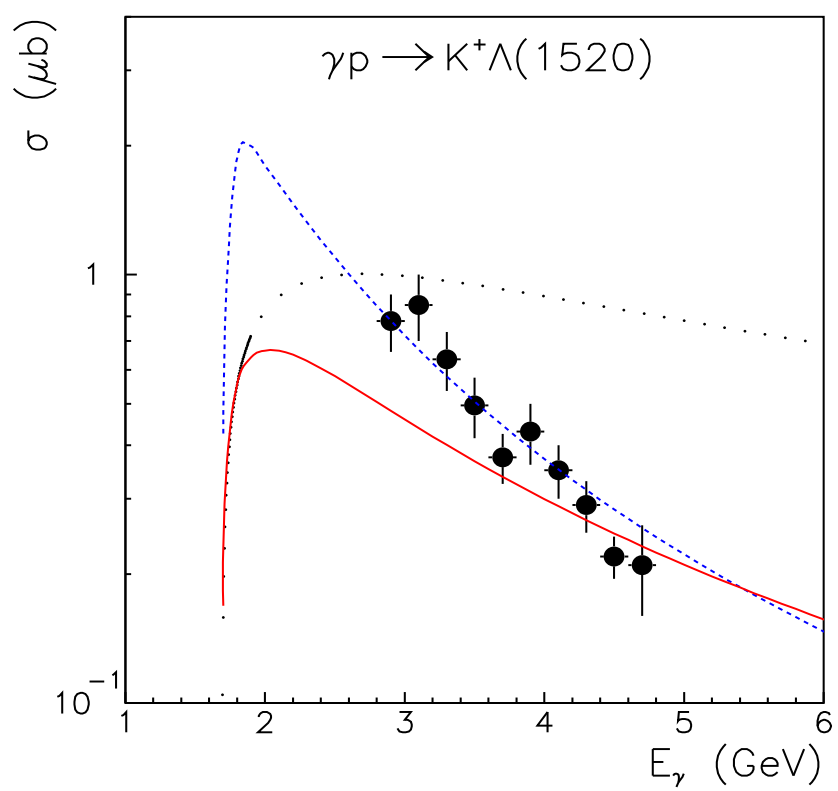

Fig. 10. The cross section for the reaction $\gamma p \rightarrow K^{+} \Lambda(1520)$. The dotted line is the result obtained with constant reaction amplitude. The dashed line shows the calculation with $K^{*}$ meson exchange without form factor and the solid line is the result obtained with form factor. The solid circles show data from Ref. [6].

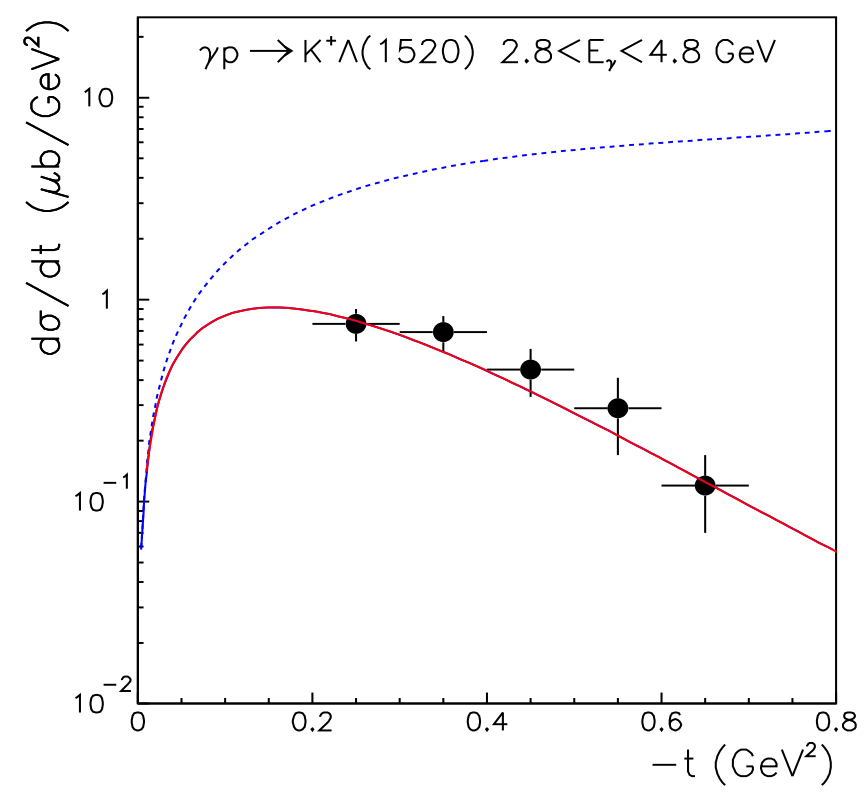

Fig. 11. The $\gamma p \rightarrow K^{+} \Lambda(1520)$ differential cross section as a function of $-t$. The curves are results of model calculations with $K^{*}$ meson exchange without form factor (dashed) and with form factor (solid). The data are from Ref. [6].

troduce absorptive corrections $[53,57,58]$ that allow one to reproduce the $t$-dependence phenomenologically.

The solid lines in Figs. 10 and 11 show results for $\gamma p \rightarrow$ $K^{+} \Lambda(1520)$ calculated with the inclusion of the form factor $F(t)=\exp (3 \mathrm{t})$ for each helicity amplitude in Eq. (12). Now the differential cross section is well reproduced by the model. However, at the same time the description of the integrated cross sections deteriorates somewhat. In order to achieve a better overall description of the experiment one could either readjust the $K^{*}$ meson exchange trajectory or assume that the form factor depends also on the energy. The latter case is more natural since the data generally indicate that the slope of the $t$ dependence depends on energy. Experimental results on the energy dependence of the slope of the $t$-dependence for different photoproduction reactions are reviewed in Refs. [59-61]. Anyway, for our purpose the semiquantitative description of the data on $\gamma p \rightarrow K^{+} \Lambda(1520)$ as given by the solid line is sufficient and, therefore, we refrain from exploring further options at this stage.

Once the amplitude for $\gamma p \rightarrow K^{+} \Lambda(1520)$ is established we can then construct the reaction amplitude $\mathcal{M}$ for $\gamma p \rightarrow K^{+} K^{-} p$. It is given by [62]

$$
\mathcal{M}\left(s, t_{1}, \theta, \phi\right)=\sum_{\lambda_{2}} M_{\lambda_{2} \lambda_{b}}^{\lambda_{1} \lambda_{a}}\left(s, t_{1}\right) G_{2}\left(s_{2}\right) M_{\lambda_{c} \lambda_{d}}^{\lambda_{2}}(\theta, \phi)
$$

where $M_{\lambda_{2} \lambda_{b}}^{\lambda_{1} \lambda_{a}}$ is the production helicity amplitude given by Eq. (12) and $M_{\lambda_{c} \lambda_{d}}^{\lambda_{2}}$ is the amplitude corresponding to the decay of the resonant state 2 with helicity $\lambda_{2}$ into the system $c d$ with helicities $\lambda_{c}$ and $\lambda_{d}$, respectively. Furthermore, $G_{2}$ is the propagator of the resonant state and $s_{2}$ is the invariant mass of the $2 \rightarrow c d$ decay products. The propagator is parametrized in 
a Breit-Wigner form with an energy dependent width as proposed by Jackson [63],

$$
G_{2}\left(s_{2}\right)=\frac{m_{2} \Gamma\left(\sqrt{s_{2}}\right)}{s_{2}-m_{2}^{2}+i m_{2} \Gamma\left(\sqrt{s_{2}}\right)},
$$

where $m_{2}$ is the resonance mass and the energy variation of the width is given by

$$
\Gamma\left(\sqrt{s_{2}}\right)=\Gamma_{0}\left[\frac{\lambda^{1 / 2}\left(s_{2}, m_{c}^{2}, m_{d}^{2}\right)}{\lambda^{1 / 2}\left(m_{2}^{2}, m_{c}^{2}, m_{d}^{2}\right)}\right]^{2 l+1} \frac{\rho\left(\sqrt{s_{2}}\right)}{\rho\left(m_{2}\right)} .
$$

In the latter formula $l$ is the orbital angular momentum of the $2 \rightarrow c d$ decay and $\Gamma_{0}$ is the width at $\sqrt{s_{2}}=m_{2}$, while $\rho\left(\sqrt{s_{2}}\right)$ is a factor varying slowly with energy given by Glashow and Rosenfeld as [64]

$$
\rho\left(\sqrt{s_{2}}\right)=\frac{1}{\sqrt{s_{2}}}\left[X^{2}+\frac{\lambda\left(s_{2}, m_{c}^{2}, m_{d}^{2}\right)}{4 s_{2}}\right]^{-l}
$$

with the parameter $X=350 \mathrm{MeV}$ fixed from a fit of the baryonic resonances in the context of unitary symmetry.

\section{References}

1. T. Nakano et al., Phys. Rev. Lett. 91, 012002 (2003) [arXiv:hepex/0301020].

2. S. Stepanyan et al., Phys. Rev. Lett. 91, 252001 (2003) [arXiv:hep-ex/0307018].

3. J. Barth et al. [SAPHIR Collaboration], Phys. Lett. B 572 (2003) 127 [arXiv:hep-ex/0307083].

4. V. Kubarovsky et al., Phys. Rev. Lett. 92, 032001 (2004) [arXiv:hep-ex/0311046].

5. Particle Data Group, Phys. Lett. B 592, 1 (2004).

6. D.P. Barber et al., Z. Phys. C 7, 17 (1980).

7. A.M. Boyarski et al., Phys. Lett. 34 B, 547 (1971).

8. D.V. Bugg, Phys. Rept. 397, 257 (2004) [arXiv:hep-ex/0412045].

9. C. Amsler and N.A. Törnqvist, Phys. Rept. 389, 61 (2004).

10. V. Baru et al., Phys. Lett. B 586, 53 (2004) [arXiv:hep$\mathrm{ph} / 0308129]$.

11. E. Byckling and K. Kajyntie, Particle Kinemtics, John Willey and Sons, London (1973).

12. S.D. Drell, Rev. Mod. Phys. 33, 458 (1961).

13. P. Söding, Phys. Lett 19, 702 (1965).

14. A.S. Krass, Phys. Rev. 159, 1496 (1967).

15. J. Ballam et al., Phys. Rev. D 5, 545 (1972).

16. R. Erbe et al., Phys. Rev. 175, 1669 (1968).

17. W. Roberts, Phys. Rev. C 70 (2004) 065201 [arXiv:nuclth/0408034].

18. L. Roca, E. Oset, and H. Toki, arXiv:hep-ph/0411155.

19. Y. Oh, K. Nakayama, and T.-S.H. Lee, Phys. Rept. 423, 49 (2006) [arXiv:hep-ph/0412363].

20. S. Nam, A. Hosaka and H.C. Kim, Genshikaku Kenkyu 49, 53 (2005) [arXiv:hep-ph/0502143].

21. S. Nam, A. Hosaka and H.C. Kim, arXiv:hep-ph/0505005.

22. V.Yu. Grishina, L.A. Kondratyuk, W. Cassing, M. Mirazita and P. Rossi, Eur. Phys. J. A 25, 141 (2005) [arXiv:nucl-th/0506053].

23. A.I. Titov, B. Kämpfer, S. Date and Y. Ohashi, Phys. Rev. C 72, 035206 (2005) [arXiv:nucl-th/0506072].

24. E. Ferrari and F. Selleri, Phys. Rev. Lett. 7, 387 (1961).

25. T. deForest and J. D. Walecka, Adv. Phys. 15, 1 (1966).
26. H. Haberzettl, Phys. Rev. C 562041 (1997).

27. A. I. Titov, H. Ejiri, H. Haberzettl, and K.Nakayama, Phys. Rev. C 71035203 (2005).

28. C. Hanhart, Phys. Rept. 397, 155 (2004).

29. V.B. Berestetsky and I.Ya. Pomeranchuk, Nucl. Phys. 22, 629 (1961)

30. E. Ferrari and F. Selleri, Suppl. Nuovo Cim. 26, 451 (1962).

31. A. Sibirtsev, Phys. Lett. B 359, 29 (1995).

32. A.A. Sibirtsev, Nucl. Phys. A 604, 455 (1996).

33. A. Sibirtsev, W. Cassing, and C.M. Ko, Z. Phys. A 358, 101 (1997) [arXiv:nucl-th/9612040].

34. G. Höhler, in Landolt-Börnstein, New Series, Vol. 9 b2 ed. H. Schopper (Springer, Berlin, 1983).

35. R. Büttgen, K. Holinde, A. Müller-Groeling, J. Speth and P. Wyborny, Nucl. Phys. A 506, 586 (1990).

36. M. Hoffmann, J.W. Durso, K. Holinde, B.C. Pearce and J. Speth, Nucl. Phys. A 593, 341 (1995).

37. V. Flaminio, I.F. Graf, J.D. Hansen, W.G. Moorhead and D.R.O. Morrison, Compilation of Cross Sections, $K^{-}$and $K^{+}$Induced reactions, Preprint CERN-HERA 79-02 (1979).

38. A. Sibirtsev, J. Haidenbauer, S. Krewald and Ulf-G. Meißner, Phys. Lett. B 599, 230 (2004) [arXiv:hep-ph/0405099].

39. V.V. Barmin et al., Phys. Atom. Nucl. 66, 1715 (2003); Yad. Fiz. 66, 1763 (2003) [arXiv:hep-ex/0304040].

40. A. Sibirtsev, J. Haidenbauer, S. Krewald and Ulf-G. Meißner, Eur. Phys. J. A 23, 491 (2005) [arXiv:nucl-th/0407011].

41. G.P. Gopal et al., Nucl. Phys. B 119 362, (1977).

42. A.D Martin and G.G. Ross, Nucl. Phys. B 16, 479 (1970).

43. C.J. Adams et al., Nucl. Phys. B 9654 (1975).

44. T.S. Mast et al., Phys. Rev. D 1413 (1976).

45. M.B. Watson, M. Ferro-Luzzi and Robert D. Tripp, Phys. Rev. 131, 2248 (1963).

46. A. Baldini, V. Flaminio, W.G. Moorhead and D.R.O. Morrison, Landolt-Börnstein, New Series, I/12b, 365 (1983).

47. G.F. Chew and F.E. Low, Phys. Rev. 113, 1640 (1959).

48. M. Ostrick, private communication; J. Barth et al., Eur. Phys. J. A 17 (2003) 269.

49. SAPHIR Collaboration, in preparation.

50. V. Kubarovsky et al., hep-ex/0605001.

51. R. de Vita and V. Kubarovsky, private communication.

52. K. Hicks, private communication.

53. A.C. Irving and R.P. Worden, Phys. Rept. 34C, 119 (1977).

54. A.B. Kaidalov and B.M. Karnakov, Sov. J. Nucl. Phys. 7, 111 (1968).

55. A.A. Zayats, Sov. J. Nucl. Phys. 16, 597 (1973).

56. A.P. Szczepaniak and M. Swat, Phys. Lett. B 516, 72 (2001) [arXiv:hep-ph/0105329].

57. K. Gottfried and J.D. Jackson, Nuovo Cim. 34, 736 (1964).

58. N. Levy, W. Majerotto and B.J. Read, Nucl. Phys B 55, 493 (1973).

59. A. Sibirtsev, K. Tsushima and S. Krewald, Phys. Rev. C 67, 055201 (2003) [arXiv:nucl-th/0301015].

60. A. Sibirtsev, S. Krewald and A.W. Thomas, J. Phys. G 30, 1427 (2004).

61. A. Sibirtsev, Ulf-G. Meißner, and A.W. Thomas, Phys. Rev. D 71 (2005) 094011 [arXiv:hep-ph/0503276].

62. P.D.B. Collins, An introduction to Regge theory and high energy physics, Cambridge University, 112 (1977).

63. J.D. Jackson, Il Nuovo Cim., 34, 1644 (1964).

64. S.L. Glashow and A.H. Rosenfeld, Phys. Rev. Lett. 10, 192 (1963). 